\title{
How much medium do you use for cell culture? Medium volume influences mineralization and osteoclastogenesis in vitro
}

\author{
YOSHITAKA YOSHIMURA $^{1}$, TAKASHI KIKUIRI ${ }^{2}$, TOMOKAZU HASEGAWA ${ }^{3}$, \\ MINO MATSUNO $^{1}$, HAJIME MINAMIKAWA ${ }^{1}$, YOSHIAKI DEYAMA ${ }^{1}$ and KUNIAKI SUZUKI ${ }^{1}$ \\ Departments of ${ }^{1}$ Molecular Cell Pharmacology and ${ }^{2}$ Pediatric Dentistry, Hokkaido University Graduate \\ School of Dental Medicine, Kita-ku, Sapporo, Hokkaido 060-8586; ${ }^{3}$ Department of Pediatric Dentistry, \\ Faculty of Dentistry, Tokushima University, Tokushima, Tokushima 770-8504, Japan
}

Received January 22, 2016; Accepted February 15, 2017

DOI: $10.3892 / \mathrm{mmr} .2017 .6611$

\begin{abstract}
Bone is maintained by a balance between bone formation and resorption. This remodeling is controlled by a wide variety of systemic and local factors including hormones, cytokines and mechanical stresses. The present in vitro study examined the impact of medium volume, using $0.4,0.6,0.8$, $1.0,1.5$ and $2.0 \mathrm{ml} /$ well in a 24 -well plate, on the differentiation of osteoblasts and osteoclasts. There were no differences in the alkaline phosphatase activity of osteoblasts amongst the groups; however, the area of mineral deposition was decreased in a media volume-dependent manner. A co-culture of osteoblastic cells with bone marrow cells revealed a reduction in the total number of osteoclastic tartrate-resistant acid phosphatase (TRAP)-positive multinuclear cells ( $\geq 2$ nuclei), whereas the formation of large osteoclastic TRAP-positive multinuclear cells ( $\geq 8$ nuclei) was increased, in a media volume-dependent manner. There were also no differences in receptor activator of nuclear factor- $\kappa \mathrm{B}$ ligand mRNA and total osteoprotegerin (OPG) protein expression levels amongst the groups, however the concentration of OPG decreased in a media volume-dependent manner. In conclusion, the present study demonstrated that the suppression of mineralization in osteoblastic cells and the stimulation of osteoclast fusion are dependent on the medium volume, indicating that media volume is an important factor in in vitro cell culture systems.
\end{abstract}

\section{Introduction}

How much medium should be used for cell culture? Some companies provide a recommended media volume for culture dishes, plates and flasks, however, there are few reports

Correspondence to: Dr Yoshitaka Yoshimura, Department of Molecular Cell Pharmacology, Hokkaido University Graduate School of Dental Medicine, Kita 13 Nishi 7, Kita-ku, Sapporo, Hokkaido 060-8586, Japan

E-mail: yoshi@den.hokudai.ac.jp

Key words: medium volume, mineralization, osteoclastogenesis, osteoblast, osteoclast investigating the ideal media volume for individual wells, dishes and flasks. The optimal medium volume for the culture of embryos (1,2), renal epithelial cells (3) and chondrocytes (4-6) has been reported, and it has been recognized that cell proli feration and differentiation are largely influenced by culture conditions, such as cell density and autocrine and/or paracrine regulators. In a study investigating in vitro embryonic development, the volume of micro-drop culture medium and the density of embryos were important factors (2). Another study demonstrated that cell viability was influenced by the media glucose content, and with increasing volumes of culture medium there are increasing amounts of glucose $(3,5)$. Furthermore, the rates of lactate reuptake appear to be highly dependent on the culture medium volume, indicating there may be a volume-induced stimulation of oxidative lactate metabolism (3). In addition, the concentration of oxygen in the medium has been demonstrated to decrease in line with increasing medium depth $(6,7)$. Collectively, this indicates that the medium volume may impact a variety of cell culture factors.

Bone homeostasis is maintained via a balance between osteoblastic bone formation and osteoclastic resorption. This remodeling is controlled by a wide variety of systemic and local factors including hormones, cytokines and mechanical stresses. Osteoblasts undergo proliferation, matrix maturation and extracellular matrix mineralization (8). During this process, osteoblasts synthesize extracellular matrix components including type I collagen. Notably, the activity of osteoblast alkaline phosphatase (ALP) increases upon reaching confluence in culture $(9,10)$. ALP hydrolyzes substrates, and the extracellular matrix is subsequently mineralized, which occurs by increasing the local calcium phosphate concentration. ALP activity has therefore been used as an important indicator of bone formation $(11,12)$. Osteoblastic MC3T3-E1 cells undergo a process of proliferation and differentiation, and then produce mineralized nodules (13). Osteoclasts are derived from hematopoietic cells and are regulated by various cytokines and hormones including interleukin (IL)-1, IL-6 and parathyroid hormone (14). It was previously reported that macrophage colony-stimulating factor and receptor activator of nuclear factor- $\kappa \mathrm{B}$ (RANK) ligand (RANKL) are necessary and sufficient for osteoclast differentiation $(15,16)$. Osteoprotegerin $(\mathrm{OPG})$ is the decoy receptor for RANKL and inhibits RANKL-RANK signaling (17). 
Osteoblasts express RANKL and OPG, and thus coordinate osteoclast differentiation and bone resorption $(18,19)$. In vitro, osteoclast formation may be induced using a co-culture of bone marrow and stromal cells (20). Cellular contact is essential for osteoclastogenesis (21).

Further clarification on the importance of medium volume in osteoblastic and osteoclastic cell culture systems in vitro is required. Therefore, the present in vitro study examined the impact of medium volume on the mineral deposition of osteoblasts and differentiation of osteoclastic precursor cells. The results indicated that the suppression of mineralization in osteoblastic cells and stimulation of the fusion process in osteoclasts is influenced by the medium volume.

\section{Materials and methods}

Osteoblast cell culture. To investigate in vitro mineralization, osteoblastic MC3T3-E1 cells were donated by Dr H. Kodama (Ohu University, Kōriyama, Japan) and were cultured in fresh $\alpha$-minimum essential medium ( $\alpha$-MEM; Wako Pure Chemical Industries, Ltd., Osaka, Japan) supplemented with $10 \%$ fetal bovine serum (FBS; Invitrogen; Thermo Fisher Scientific, Inc., Waltham, MA, USA), 2 mM L-alanyl-L-glutamine (Wako Pure Chemical Industries, Ltd., ), $284 \mu \mathrm{M}$ L-ascorbic acid 2-phosphate (Sigma-Aldrich; Merck KGaA, Darmstadt, Germany) and $66.7 \mathrm{mg} / \mathrm{ml}$ kanamycin sulfate (Meiji Seika Kaisha, Ltd., Tokyo, Japan) in 24- and 12-well plates. Cells were cultured in $0.4,0.6,0.8,1.0,1.5$ or $2.0 \mathrm{ml}$ medium, and the culture medium was changed every other day. All cultures were maintained at $37^{\circ} \mathrm{C}$ in a humidified atmosphere with $5 \%$ $\mathrm{CO}_{2}$. After culture for 30 days, the cells were fixed with $10 \%$ neutral formalin (Wako Pure Chemical Industries, Ltd.) and subjected to von Kossa staining as previously described (13). Briefly, a solution of $1 \%$ aqueous silver nitrate was added to each dish under direct light for $15 \mathrm{~min}$.

Measurement of ALP activity. After culture for 30 days, ALP activity was assayed using the method described by Suzuki et al (22), which is based on the method developed by Bessey et al (23). Briefly, MC3T3-E1 cell homogenates were prepared by ultrasonication and preincubated at $37^{\circ} \mathrm{C}$ in a reaction mixture $(0.5 \mathrm{ml})$ containing $50 \mathrm{mM}$ sodium carbonate (pH 9.7), $25 \mathrm{mM}$ sucrose and $1 \mathrm{mM} \mathrm{MgCl}_{2}$ with or without $5 \mathrm{mM}$ levamisole. Assays were initiated by adding $p$-nitrophenylphosphate $(p \mathrm{NPP})$ substrate $(0.1 \mathrm{ml})$ to the reaction mixture to a final concentration of $20 \mathrm{mM}$. After $15 \mathrm{~min}$ at $37^{\circ} \mathrm{C}, 0.5 \mathrm{ml}$ reaction mixture was added to $1.5 \mathrm{ml} 0.6 \mathrm{M}$ $\mathrm{NaOH}$ solution for color development. Hydrolysis of $p$ NPP was measured colorimetrically at $420 \mathrm{~nm}$ using a microplate reader. The protein concentration of MC3T3-E1 cell homo genates was determined using a DC Protein Assay kit (Bio-Rad Laboratories, Inc., Hercules, CA, USA).

Osteoclastcell culture. A total of 20 C57BL/6J Jms Slc male mice were obtained from Sankyo Labo Service Corporation, Inc., (Tokyo, Japan). All animal experiments were performed under an institutionally approved protocol for use of animal research at Hokkaido University (no. 10-0070). Mice were kept in the animal facility at a temperature of $23^{\circ} \mathrm{C}$ and controlled humidity, under a $12 \mathrm{~h}$ light/dark cycle, and had free access to food and water. To investigate in vitro osteoclastogenesis, bone marrow cells were collected by flushing femoral shafts of 4-week-old mice were sacrificed with $\mathrm{CO}_{2}$, using a 26-gauge sterile needle. Bone marrow cells $\left(1 \times 10^{5}\right.$ cells/well) were co-cultured with proliferating MC3T3-E1 cells, using fresh $\alpha$-MEM supplemented with $10 \%$ FBS, 2 mM L-alanyl-L-glutamine, $284 \mu \mathrm{M}$ L-ascorbic acid 2-phosphate, $100 \mathrm{nM}$ dexamethasone, $10 \mathrm{nM}$ 1-alpha, 25-dihydroxyvitamin $\mathrm{D}_{3}$ (Sigma-Aldrich; Merck $\mathrm{KGaA}$ ) and $66.7 \mu \mathrm{g} / \mathrm{ml}$ kanamycin sulfate in a 24 -well plate. Cells were cultured in $0.4,0.6,0.8,1.0,1.5$ or $2.0 \mathrm{ml}$ medium. Following co-culture for 6 days, cells were fixed and stained for tartrate-resistant acid phosphatase (TRAP) activity as described previously (24). Briefly, TRAP staining solution contained acetate buffer ( $\mathrm{pH}$ 5.0), naphthol AS-MX phosphate and red violet LB (all from Sigma-Aldrich; Merck KGaA), in the presence of $50 \mathrm{mM}$ sodium tartrate (Wako Pure Chemical Industries, Ltd.). TRAP-positive multinuclear cells were considered to be osteoclasts. The numbers of TRAP-positive multinuclear cells were expressed as the mean \pm standard deviation (SD) of triplicate cultures.

Semi-quantitative measurement of RANKL mRNA. MC3T3-E1 cells were co-cultured as aforementioned for 6 days. Total RNA was isolated using TRIzol (Invitrogen; Thermo Fisher Scientific, Inc.) according to the manufacturer's protocol. First strand cDNA was synthesized using a Revatra Ace FSK-101 kit (Toyobo Co.,Ltd., Osaka, Japan). The reaction buffer contained the following components: $50 \mathrm{mM}$ Tri-HCl (pH 7.5), $100 \mathrm{mM}$ $\mathrm{NaCl}, 0.1 \mathrm{mM}$ EDTA, $10 \mathrm{mM}$ dithioerythritol, $0.01 \%$ Nonidet P-40 and 50\% glycerol. The reaction conditions for the reverse transcription (RT) procedure were as follows: Annealing at $30^{\circ} \mathrm{C}$ for $10 \mathrm{~min}$, extension at $42^{\circ} \mathrm{C}$ for $20 \mathrm{~min}$, denaturation at $99^{\circ} \mathrm{C}$ for $5 \mathrm{~min}$ followed by cooling at $4^{\circ} \mathrm{C}$. KOD-Dash DNA polymerase reaction mixture (Toyobo Co., Ltd.) was used for amplification. Polymerase chain reaction (PCR) amplification was performed with a GeneAmp PCR system 9700 (Applied Biosystems; Thermo Fisher Scientific, Inc.) using the following thermocycling conditions: 23 cycles for GAPDH and 30 cycles for RANKL of $94^{\circ} \mathrm{C}$ for $30 \mathrm{sec}$, annealing at $60^{\circ} \mathrm{C}$ for $2 \mathrm{sec}$ and extension at $72^{\circ} \mathrm{C}$ for $30 \mathrm{sec}$. The first cycle was conducted at $94^{\circ} \mathrm{C}$ for $10 \mathrm{~min}$ and the final extension cycle at $72^{\circ} \mathrm{C}$ for $10 \mathrm{~min}$. The following primers were used: RANKL, forward 5'-TATGATGGAAGGCTCATGGT-3' and reverse 5'-TGT CCTGAACTTTGAAAGCC-3'; GAPDH, forward 5'-CGG AGTCAACGGATTTGGTCGTAT-3' and reverse 5'-AGCCT TCTCCATGGTGGTGAAGAC-3'. Amplification products were separated on a $1 \%$ agarose gel and stained with ethidium bromide. Densitometry was performed using Image J software version 1.48a (National Institutes of Health, Bethesda, MD, USA). GAPDH was used as the housekeeping gene for normalizing expression values (25).

Measurement of OPG content. OPG content recovered from the osteoclast co-culture system conditioned medium was measured using a mouse OPG Analyza ELISA kit (catalog no. 10047; Bio-Techne Co, Minneapolis, MN, USA), according to the manufacturer's protocol. In brief, standard solutions and conditioned medium were plated in a 96-well microplate pre-coated with monoclonal antibody specific to mouse OPG and incubated for $2 \mathrm{~h}$ at room temperature. After washing, 


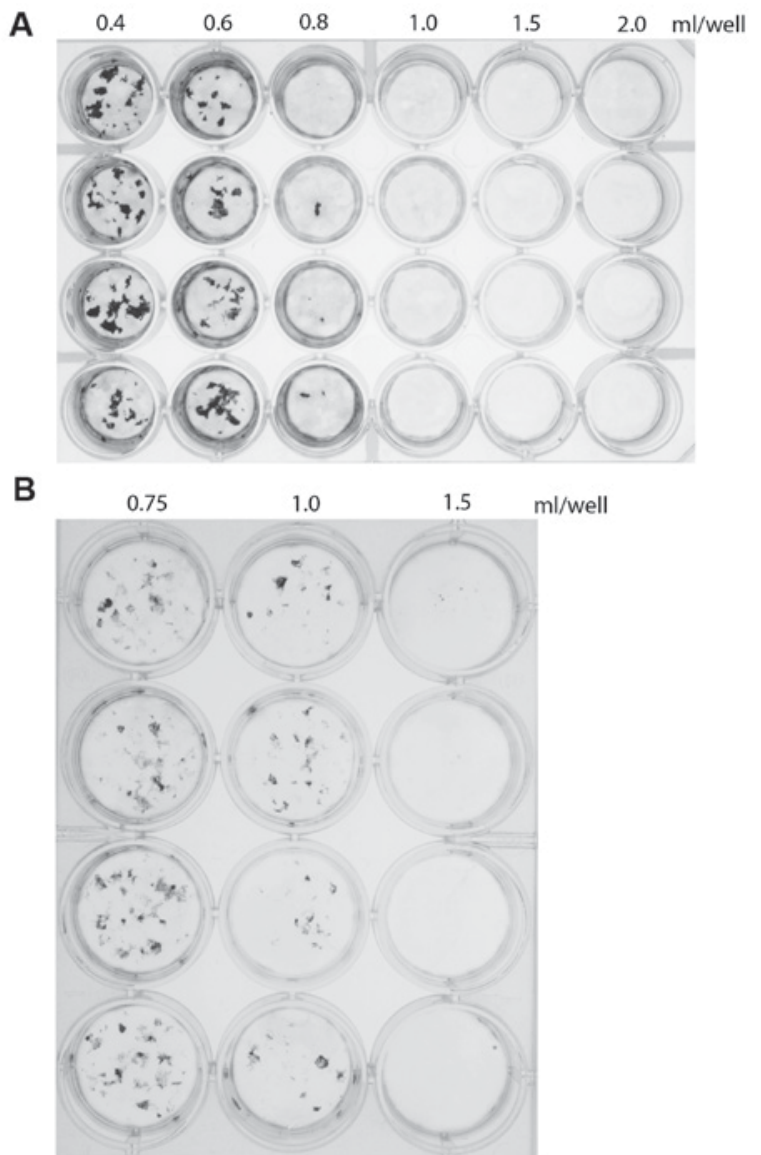

Figure 1. Effects of medium volume on the area of mineral deposition in osteoblastic MC3T3-E1 cells. Following a 30-day culture, cells were subjected to von Kossa staining. (A) Cells were cultured in 0.4, 0.6, 0.8, 1.0, 1.5 or $2.0 \mathrm{ml} /$ well in a 24 -well plate. (B) Cells were cultured in $0.75,1.0$ or $1.5 \mathrm{ml} /$ well in a 12 -well plate.

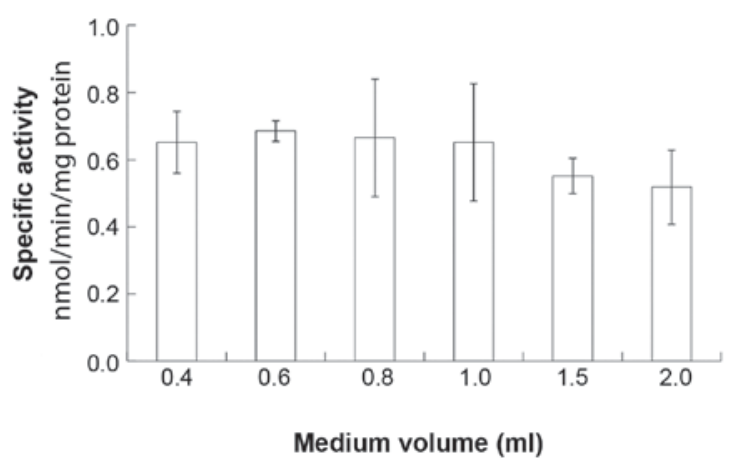

Figure 2. Effects of medium volume on ALP activity in osteoblastic MC3T3-E1 cells. Following 30 day culture, ALP activity of osteoblasts did not differ when cultured in $0.4,0.6,0.8,1.0,1.5$ or $2.0 \mathrm{ml} /$ well. Data are presented as the mean \pm standard deviation $(\mathrm{n}=3)$. ALP, alkaline phosphatase.

a peroxidase-labeled polyclonal antibody specific to OPG was added to each well and incubated for $2 \mathrm{~h}$. Subsequently, unbound antibody-enzyme was removed by washing and the substrate was added to the wells. Following incubation for $30 \mathrm{~min}$ at room temperature, the absorbance was measured at a wavelength of $450 \mathrm{~nm}$ using a microplate reader (Bio-Rad Laboratories, Inc.).
Statistical analysis. Values were expressed as the mean \pm SD. Overall comparisons were performed using a two-way analysis of variance followed by the Bonferroni's post hoc test. $\mathrm{P}<0.05$ was considered to indicate a statistically significant difference.

\section{Results}

The present study examined the impact of culture medium volume on the in vitro differentiation of osteoblastic MC3T3-E1 cells, using $0.4,0.6,0.81 .0,1.5$ or $2.0 \mathrm{ml} /$ well culture medium, in a 24 -well plate. Mineral deposition was measured after 30 days of culture. The results indicated that the area of mineral deposition was inversely proportional to the volume of medium (Fig. 1A). To verify these results, mineral deposition was investigated using a 12 -well plate with $0.75,1.0$ or $1.5 \mathrm{ml} /$ well. Once again, the area of mineral deposition area was inversely proportional to the medium volume (Fig. 1B), confirming the results obtained in the initial experiment. Notably, there was no significant difference in the osteoblastic ALP activity amongst the groups (P>0.05; Fig. 2).

Co-culture of osteoblastic MC3T3-E1 cells with C57BL/6 mouse-derived bone marrow cells was performed, and the number of TRAP-positive cells was quantified. The total number of TRAP-positive multinuclear cells was reduced in a medium volume-dependent manner (Fig. 3A), whereas the formation of large osteoclastic TRAP-positive multinuclear cells ( $\geq 8$ nuclei) was increased in a medium volume-dependent manner (Fig. 3B). Furthermore, the ratio of osteoclasts with 2-7 nuclei to osteoclasts with $\geq 8$ nuclei decreased with increasing media volume (Fig. 3C).

To investigate the impact of media volume on RANKL mRNA levels, the expression of RANKL mRNA was semi-quantified using RT-PCR. The expression of RANKL mRNA did not appear to differ between groups (Fig. 4). ELISA was used to investigate the effects of medium volume on OPG content. The concentration of OPG was inversely proportional to the volume of media $(\mathrm{P}<0.05$; Fig. $5 \mathrm{~A})$, however the total content of OPG did not differ between groups $(\mathrm{P}>0.05$; Fig. 5B).

\section{Discussion}

The results indicated that there was a negative association between the volume of media and the occurrence of mine ralization in cultured osteoblasts (Fig. 1). Previous research has indicated that cell viability is influenced by glucose availability, and increasing volumes of culture medium therefore contain increasing amounts of glucose $(3,5)$. However, the area of mineral deposition was inversely proportional to medium volume. Although the total content of cytokines would most likely be the same within this culture system, the different media volumes result in concentration or dilution of the total cytokine content, and the cytokine concentrations were therefore inversely proportional to the medium volume. This result indicates that osteoblast mineralization may be promoted in an autocrine manner, regardless of the glucose content. Previous studies have also demonstrated that oxygen concentrations in the culture media decrease in line with increasing medium 
A

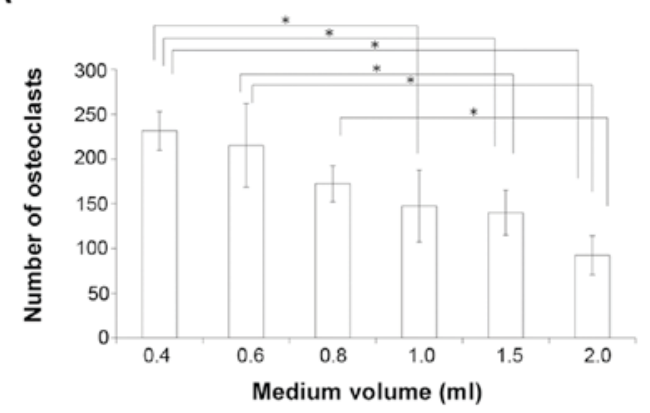

B

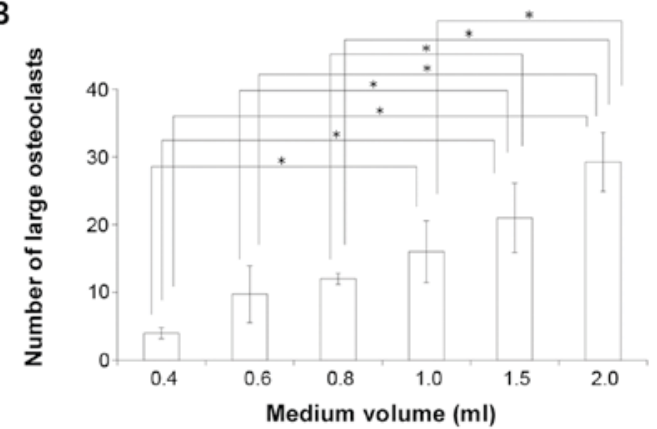

C

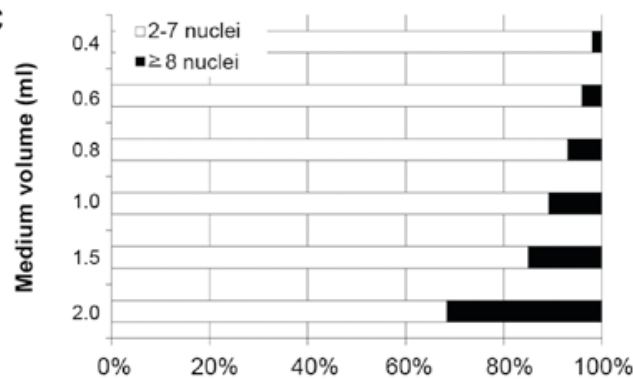

Figure 3. Effects of medium volume on the formation of osteoclastic TRAP-positive multinuclear cells in a co-culture system. (A) The number of osteoclastic TRAP-positive multinuclear cells containing $\geq 2$ nuclei. (B) The number of large TRAP-positive multinuclear cells ( $\geq 8$ nuclei). (C) The ratio of osteoclasts with 2-7 nuclei cells and $\geq 8$ nuclei cells. Data are presented as the mean \pm standard deviation $(\mathrm{n}=6) .{ }^{*} \mathrm{P}<0.05$, with comparisons indicated by brackets. TRAP, tartrate-resistant acid phosphatase.

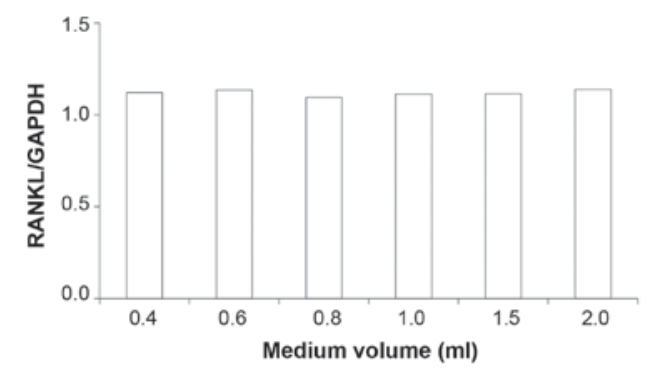

Figure 4. Effects of medium volume on RANKL mRNA expression in MC3T3-E1 cells. The mRNA of RANKL and GAPDH were determined by semi-quantitative reverse transcription polymerase chain reaction. Relative expression of RANKL mRNA to that of GAPDH mRNA is indicated. Assays were repeated three times and representative experiments are demonstrated as relative densitometric units. RANKL, receptor activator of NF- $\kappa B$ ligand.

depth $(6,7)$. The media volumes of $0.4,0.6,0.8,1.0,1.5$ and $2.0 \mathrm{ml}$ in a 24 -well plate $\left(2 \mathrm{~cm}^{2} /\right.$ well $)$ correspond to media depths of 2, 3, 4, 5, 7.5 and $10 \mathrm{~mm}$, respectively. The concentration of oxygen in the medium affects matrix synthesis
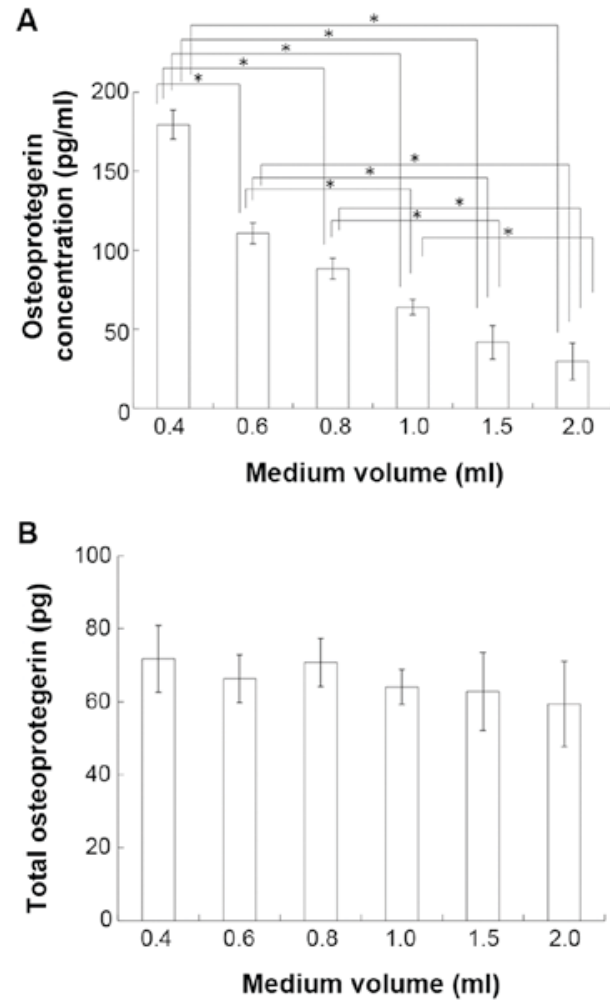

Figure 5. Effects of medium volume on OPG production in MC3T3-E1 cells. The OPG content of conditioned media was measured by ELISA. (A) OPG concentration in $0.4,0.6,0.8,1.0,1.5$ and $2.0 \mathrm{ml} / \mathrm{well}$. (B) Total OPG content in $0.4,0.6,0.8,1.0,1.5$ and $2.0 \mathrm{ml} /$ well. Data are presented as the mean \pm standard deviation $(n=6)$. ${ }^{*} \mathrm{P}<0.05$, with comparisons indicated by brackets. OPG, osteoprotegerin

and differentiation in murine chondrogenic cell culture (6), and therefore the altered osteoblastic mineralization may be related to oxygen concentration. ALP activity has been used as an important indicator of bone formation; however, in the present study, there were no significant differences in the ALP activity of MC3T3-E1 cells among the cultures. Therefore, these results suggested that ALP activity is not influenced by these conditions.

RANKL is an essential factor in osteoclast differentiation and activation (17). Osteoblasts express RANKL and $\mathrm{OPG}$, and thus coordinate osteoclast differentiation and bone resorption $(15,26)$. Osteoclastogenesis is dependent on RANKL concentration (27); therefore, the present study employed a co-culture system with osteoblastic cells and bone marrow cells without RANKL. In this co-culture system, the total number of TRAP-positive multinuclear osteoclasts was reduced in a medium volume-dependent manner, although the formation of large TRAP-positive multinuclear osteoclasts ( $\geq 8$ nuclei) was increased, indicating that osteoclastogenesis was increased under these conditions. To investigate whether the inductive effect of medium volume on osteoclasto genesis is dependent on the amount of RANKL and/or OPG produced by osteoblasts, the amount of RANKL mRNA and the secretion of OPG into the conditioned medium were measured. OPG is the decoy receptor for RANKL, and it inhibits RANKL-RANK signaling (5). No significant impact of medium volume on RANKL mRNA expression was observed. Furthermore, there were no significant differences 
in the total amount of OPG amongst the various culture conditions; however, the concentration of OPG was inversely proportional to medium volume. This indicated that a balance between RANKL and OPG concentrations influences osteoclast fusion.

Increasing the volume of medium exerts mechanical stress, such as hydraulic pressure, on cells. Human periodontal ligament cells under hydraulic pressure display reduced OPG mRNA expression in a medium volume-dependent manner (28); although these results appear to conflict with the present study, this discrepancy may be attributable to inherent differences between human periodontal ligament cells and murine osteoblasts. A recent study demonstrated that osteoclastogenesis was accelerated by an optimal compressive force (24), where a compressive force was directly applied to the osteoclast-precursor cells, without the use of a co-culture system. Furthermore, osteoclastogenesis appears to be accelerated by increasing volumes of culture medium and a concomitant increase in nutrients, including glucose $(3,5)$. Glucose was the principal energy source required for bone degradation (29), and hypoxia-inducible factor 1 alpha was stabilized in osteoclasts, leading to osteoclast activation (30). This suggests that the differentiation of large multinuclear osteoclasts may be influenced by the glucose content, and also by low concentrations of oxygen in the medium $(6,7)$. Notably, the total number of large osteoclasts ( $\geq 8$ nuclei) was observed to increase in a medium volume-dependent manner. These results indicated that, in an in vitro system, the volume of medium may have a greater effect on promoting the osteoclast fusion process, compared with the differentiation of mononuclear osteoclasts and/or osteoclastic precursors. By contrast, the formation of 2-7 nuclei osteoclasts appeared to be reduced in a medium volume-dependent manner; this may be due to the concentrations of oxygen or of osteoclast inducible cytokines, such as IL-1 and tumor necrosis factor- $\alpha$ (TNF- $\alpha)$, which are reportedly inversely proportional to medium volume (31). This may be due to IL-1 and TNF- $\alpha$ stimulating osteoclast differentiation from mononuclear pre-osteoclasts to small osteoclasts via RANKL-RANK signaling (31).

Thus, the nutrient availability of the culture medium appears to be influenced by the medium volume, and this may directly influence osteoblast mineralization and osteoclast differentiation (3). The volume of media used in cell culture is therefore an important consideration in the culture of osteoblasts and osteoclasts, and culture systems should be optimized to ensure an optimal in vitro microenvironment is achieved.

\section{References}

1. Kito S, Iritani A and Bavister BD: Effects of volume, culture media and type of culture dish on in vitro development of hamster 1-cell embryos. Theriogenology 47: 541-548, 1997.

2. Dai SJ, Xu CL, Wang J, Sun YP and Chian RC: Effect of culture medium volume and embryo density on early mouse embryonic development: Tracking the development of the individual embryo. J Assist Reprod Genet 29: 617-623, 2012.

3. Gstraunthaler G, Seppi T and Pfaller W: Impact of culture conditions, culture media volumes, and glucose content on metabolic properties of renal epithelial cell cultures. Are renal cells in tissue culture hypoxic? Cell Physiol Biochem 9: 150-172, 1999.

4. Heywood HK, Sembi PK, Lee DA and Bader DL: Cellular utilization determines viability and matrix distribution profiles in chondrocyte-seeded alginate constructs. Tissue Eng 10: $1467-1479,2004$
5. Heywood HK, Bader DL and Lee DA: Glucose concentration and medium volume influence cell viability and glycosaminoglycan synthesis in chondrocyte-seeded alginate constructs. Tissue Eng 12: 3487-3496, 2006.

6. Oze H, Hirao M, Ebina K, Shi K, Kawato Y, Kaneshiro S, Yoshikawa $\mathrm{H}$ and Hashimoto J: Impact of medium volume and oxygen concentration in the incubator on pericellular oxygen concentration and differentiation of murine chondrogenic cell culture. In Vitro Cell Dev Biol Anim 48: 123-130, 2012.

7. Pettersen EO, Larsen LH, Ramsing NB and Ebbesen P: Pericellular oxygen depletion during ordinary tissue culturing, measured with oxygen microsensors. Cell Prolif 38: 257-267, 2005.

8. Owen TA, Aronow M, Shalhoub V, Barone LM, Wilming L, Tassinri MS, Kennedy MB, Pockwines S, Lian JB and Stein GS: Progressive development of the rat osteoblast phenotype in vitro: Reciprocal relationship in expression of genes associated with osteoblast proliferation and differentiation during formation of the bone extracellular matrix. J Cell Physiol 143: 420-430, 1990.

9. Quarles LD, Yohay DA, Lever LW, Caton R and Wenstrup RJ: Distinct proliferative and differentiated stages of murine MC3T3-E1 cells in culture: An in vitro model of osteoblast development. J Bone Miner Res 7: 683-692, 1992.

10. Deyama Y, Takeyama S, Koshikawa M, Shirai Y, Yoshimura Y, Nishikata M, Suzuki K and Matsumoto A: Osteoblast maturation suppressed osteoclastogenesis in coculture with bone marrow cells. Biochem Biophys Res Commun 274: 249-254, 2000.

11. Anderson HC: Molecular biology of matrix vesicles. Clin Orthop Relat Res 266-280, 1995.

12. Fleish H and Neuman WF: Mechanism of calcification. Role of collagen, polyphosphatases, and phosphate. Am J Physiol 200: 1296-1300, 1961

13. Deyama A, Deyama Y, Matsumoto A, Yoshimura Y, Nishikata M, Suzuki K and Totsuka Y: A low calcium environment enhances AP-1 transcription factor-mediated gene expression in the development of osteoblastic MC3T3-E1 Cells. Miner Electrolyte Metab 25: 147-160, 1999.

14. Suda T, Takahashi N and Martin TJ: Modulation of osteoclast differentiation. Endocr Rev 13: 66-80, 1992.

15. Yoshida H, Hayashi S, Kunisada T, Ogawa M, Nishikawa S, Okamura H, Sudo T, Shultz LD and Nishikawa SI: The murine mutation osteopetrosis is in the coding region of the macrophage colony stimulating factor gene. Nature 345: 442-444, 1990.

16. Lacey DL, Timms E, Tan HL, Kelley MJ, Dunstan CR, Burgess T, Elliott R, Colombero A, Elliott G, Scully S, et al: Osteoprotegerin ligand is a cytokine that regulates osteoclast differentiation and activation. Cell 93: 165-176, 1998.

17. Udagawa N, Takahashi N, Yasuda H, Mizuno A, Itoh K, Ueno Y, Shinki T, Gillespie MT, Martin TJ, Higashio K and Suda T: Osteoprotegerin produced by osteoblasts is an important regulator in osteoclast development and function. Endocrinology 141: 3478-3484, 2000.

18. Boyle WJ, Simonet WS and Lacey DL: Osteoclast differentiation and activation. Nature 423: 337-342, 2003.

19. Mochizuki A, Takami M, Kawawa T, Suzumoto R, Sasaki T, Shiba A, Tsukasaki H, Zhao B, Yasuhara R, Suzawa T, et al: Identification and characterization of the precursors committed to osteoclasts induced by TNF-related activation-induced cytokine/receptor activator of NF-kappa B ligand. J Immunol 177: 4360-4368, 2006.

20. Takahashi N, Akatsu T, Udagawa N, Sasaki T, Yamaguchi A, Moseley JM, Martin TJ and Suda T: Osteoblastic cells are involved in osteoclast formation. Endocrinology 123: 2600-2602, 1988.

21. Suda T, Udagawa N, Nakamura I, Miyaura C and Takahashi N: Modulation of osteoclast differentiation by local factors. Bone 17 (2 Suppl): 87S-91S, 1995.

22. Suzuki K, Yoshimura Y, Hisada Y and Matsumoto A: Sensitivity of intestinal alkaline phosphatase to L-homoarginine and its regulation by subunit-subunit interaction. Jpn J Pharmacol 64: 97-102, 1994.

23. Bessey OA, Lowry OH and Brock MJ: A method for the rapid determination of alkaline phosphatase with five cubic millimeters of serum. J Biol Chem 164: 321-329, 1946.

24. Hayakawa T, Yoshimura Y, Kikuiri T, Matsuno M, Hasegawa T, Fukushima K, Shibata K, Deyama Y, Suzuki K and Iida J: Optimal compressive force accelerates osteoclastogenesis in RAW264.7 cells. Mol Med Rep 12: 5879-5885, 2015. 
25. Nakai T, Yoshimura Y, Deyama Y, Suzuki K and Iida J: Mechanical stress up-regulates RANKL expression via the VEGF autocrine pathway in osteoblastic Mc3T3-E1 cells. Mol Med Rep 2: 229-234, 2009.

26. Simonet WS, Lacey DL, Dunstan CR, Kelley M, Chang MS Lüthy R, Nguyen HQ, Wooden S, Bennett L, Boone T, et al: Osteoprotegerin: A novel secreted protein involved in the regulation of bone density. Cell 89: 309-319, 1997.

27. Suzuki N, Yoshimura Y, Deyama Y, Suzuki K and Kitagawa Y: Mechanical stress directly suppresses osteoclast differentiation in RAW264.7 cells. Int J Mol Med 21: 291-296, 2008.

28. Nakao K, Goto T, Gunjigake KK, Konoo T, Kobayashi S and Yamaguchi K: Intermittent force induces high RANKL expression in human periodontal ligament cells. J Dent Res 86: 623-628, 2007.
29. Williams JP, Blair HC, McDonald JM, McKenna MA, Jordan SE, Williford J and Hardy RW: Regulation of osteoclastic bone resorption by glucose. Biochem Biophys Res Commun 235: 646-651, 1997

30. Miyauchi Y, Sato Y, Kobayashi T, Yoshida S, Mori T, Kanagawa H, Katsuyama E, Fujie A, Hao W, Miyamoto K, et al: HIF1 $\alpha$ is required for osteoclast activation by estrogen deficiency in postmenopausal osteoporosis. Proc Natl Acad Sci USA 110: 16568-16573, 2013.

31. Takayanagi H: Osteoimmunology: Shared mechanisms and crosstalk between the immune and bone systems. Nat Rev Immunol 7: 292-304, 2007. 